\title{
EEG anticipation of random high and low arousal faces and
}

\section{sounds [version 1; peer review: 1 approved, 1 not approved]}

\author{
Gian Marco Duma1', Giovanni Mento², Luca Semenzato², Patrizio E. Tressoldi (D2 \\ ${ }^{1}$ Dipartimento di Psicologia dello Sviluppo e della Socializzazione, Università di Padova, Padova, Italy \\ ${ }^{2}$ Dipartimento di Psicologia Generale, Università di Padova, Padova, Italy
}

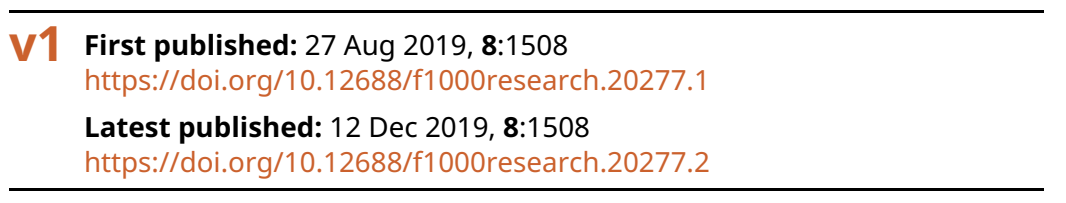

\section{Abstract}

Background: In this study, we investigated the neural correlates of the anticipatory activity of randomly presented faces and sounds of both high and low arousal level by recording EEG activity with a high spatial resolution EEG system.

Methods: We preregistered the following three hypotheses: 1) a contingent Negative Variation (CNV) difference in the amplitude voltage between auditory vs faces stimuli; 2 ) a greater amplitude voltage in the CNV, in high arousal stimuli vs low arousal stimuli, both in auditory and faces stimuli, in the temporal window from 0 to 1000 ms before the stimulus presentation; 3 ) in the time window from 0 to $1000 \mathrm{~ms}$ a sensory specific activation at the brain source level in the temporal lobe and auditory cortex before the presentation of an auditory stimulus and an activation of occipital area, dedicated to the elaboration of visual stimuli, before the presentation of faces .

Results: Using a preregistered, hypothesis-driven approach, we found no statistically significant differences in the CNV due to an overly conservative correction for multiple comparisons for the control of Type I error. By contrast, using a data-driven approach based on a machine learning algorithm (Support Vector Machine), we found a significantly larger amplitude in the occipital cluster of electrodes before the presentation of faces with respect to sounds, along with a larger amplitude in the right auditory cortex before the presentation of sounds with respect to faces. Furthermore, we found greater CNV activity in the late prestimulus interval for high vs. low-arousal sounds stimuli in the left centro-posterior scalp regions.

Conclusions: These findings, although preliminary, seem to support the hypothesis that the neurophysiological anticipatory activity of random events is specifically driven by either the sensory characteristics or the arousal level of future stimuli.

\section{Keywords}

anticipation, random events, EEG, source analysis, support vector machine

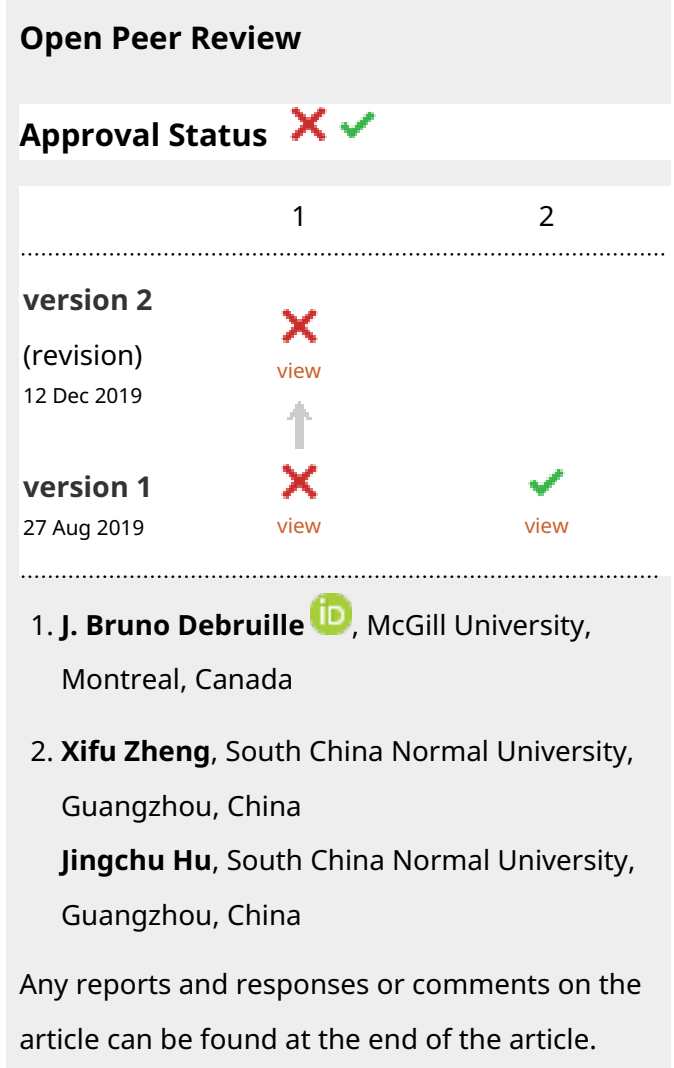


Corresponding author: Patrizio E. Tressoldi (patrizio.tressoldi@unipd.it)

Author roles: Duma GM: Conceptualization, Data Curation, Formal Analysis, Methodology, Writing - Original Draft Preparation, Writing - Review \& Editing; Mento G: Conceptualization, Formal Analysis, Investigation, Methodology, Supervision, Writing - Original Draft Preparation, Writing - Review \& Editing; Semenzato L: Methodology, Software; Tressoldi PE: Conceptualization, Supervision, Writing Review \& Editing

Competing interests: No competing interests were disclosed.

Grant information: The author(s) declared that no grants were involved in supporting this work.

Copyright: @ 2019 Duma GM et al. This is an open access article distributed under the terms of the Creative Commons Attribution License, which permits unrestricted use, distribution, and reproduction in any medium, provided the original work is properly cited.

How to cite this article: Duma GM, Mento G, Semenzato L and Tressoldi PE. EEG anticipation of random high and low arousal faces and sounds [version 1; peer review: 1 approved, 1 not approved] F1000Research 2019, 8:1508

https://doi.org/10.12688/f1000research.20277.1

First published: 27 Aug 2019, 8:1508 https://doi.org/10.12688/f1000research.20277.1 


\section{Introduction}

When we observe the world, reality is not always chaotic and unpredictable. Rather, it shows some spatiotemporal regularities. The capacity to extract these regularities is a fundamental survival function for an organism, since it allows for the finegrained, proactive control of resource allocation necessary for stimuli elaboration or action preparation. Taking advantage of these regularities, the brain building up inner models of external reality anticipating forthcoming events implementing its predictive aptitude by capitalizing on Bayesian computational architecture and, consequently, optimizing our behaviour over time (Friston, 2005; Mento, 2017; Mento \& Vallesi, 2016; Mento et al., 2015),

This account posits the brain as a mechanism that makes continuous inferences about forthcoming stimuli on the basis of conditional probabilistic computations (Chater et al., 2006; Pouget et al., 2013) accomplished by exploiting the sensory contingencies provided by the external sensory environment and the internal representation of the events.

In the last decade, neuroimaging evidence has shown that the possibility of predicting the 'what', 'when', and 'where' of forthcoming events translates into anticipatory neural activity (Mento et al., 2015; Mento et al., 2018; Mento, 2017; Mento et al., 2013). Notably, the experimental paradigms of this literature took advantage of statistically predictable stimuli, where predictive information about stimulus identity and time-space features were provided by cue presentation. A consistent finding reported in the literature is the observation of a sustained, slow event-related activity preceding the onset of predicted stimuli. This wave, known in literature as the Contingent Negative Variation (CNV; Rebert \& Tecce, 1973; Walter et al., 1964), has been interpreted as a general neural marker of anticipatory processes, including motor preparation, temporal expectancy, and temporal attention. Interestingly, the CNV is sensitive to the subjective (rather than objective) implementation of anticipatory processes (Mento et al., 2013; Trillenberg et al., 2000) in the context of temporally predictable events (Mento et al., 2015; Mento, 2017; Miniussi et al., 1999).

Recent studies explored the possibility of extending the study of anticipatory brain activity to statistically unpredictable stimuli (Duggan \& Tressoldi, 2018; Duma et al., 2017; Mossbridge et al., 2012; Radin et al., 2011), more generally defined by Mossbridge et al. (2014) as Predictive Anticipatory Activity (PPA). In our previous work (Duma et al., 2017), we investigated brain PPA phenomena in relation to the passive presentation of randomly presented car accidents compared with safe journeys. The results showed a prestimulus greater CNV negativity amplitude for trials related to car crashes from those ending with no car accidents. These results suggested that statistically unpredictable events may nevertheless engage differential anticipatory neural activity. However, since the experimental design we employed in our previous study had possible methodological limitations in the implementation of a pseudo- rather than true-random trial sorting procedure, as well as in the use of a variable anticipatory time window, we designed a new paradigm to further address any possible procedural issues.

To address any possible methodological pitfalls, in the present work we used a trial randomization procedure mediated by a true random number generator. Unlike the implementation of a pseudo-random trial sorting procedure, this method ensures that the experimental conditions presented to participants are fully randomized, so that any possible bias arising from employing a seed-based trial selection can occur, including casual repetition of the same condition in many consecutive trials. Indeed, this issue could result in the presence of possible biases conditioning both participants' behavioural and neural correlates. Furthermore, to avoid any possible bias due to implicit temporal expectancy of stimuli induced by a variable prestimulus interval (i.e., hazard function (Mento et al., 2015)), we purposely used a fixed prestimulus time window. As a second goal of the present study, we aimed at extending the knowledge about the nature of PPA phenomena, with the specific interest of investigating whether such phenomena are aspecific (i.e., independent from stimuli and task) or specific (i.e., different from stimulus type and task). To this end, in the current study, a group of healthy volunteers were asked to predict either the sensory category (i.e., visual or auditory) or the emotional meaning (i.e., low or high arousal level) of randomly delivered target stimuli in the context of both a passive and an active experimental task. Finally, in line with the methodological guidelines for increasing the reproducibility of scientific findings, the methodological procedure of this study was pre-registered.

\section{Methods}

Study pre-registration

The method of this study was registered before data collection and is available here: https://osf.io/uf59a

\section{Participants}

Thirty healthy participants recruited from graduate and undergraduate students of Padova University, with normal or corrected-to-normal vision participated in the experiment. Due to the high level of noise artefact, data from two participants were removed from the analysis. All participants received $10 €$ for their participation in the study. The study was reviewed and approved by the ethical committee of the School of Psychology of the University of Padua (Protocol No. 2278). Before the study, participants signed an informed consent in accordance with the principles expressed in the Declaration of Helsinki.

The sample size of this study was reduced with respect to what was declared in the pre-registration due to laboratory use limitation. For this reason, we were only able to collect data from 30 participants instead of the 36 declared. This reduction clearly impacted the statistical power of our planned data analyses.

\section{Experimental design}

In the present experiment, we used two sensory categories of stimuli (i.e., visual and auditory), which were extracted from two standardized international archives. Visual stimuli consisted 
of pictures of 28 faces extracted from the NIMSTIM archive (Tottenham et al., 2009), whereas auditory stimuli consisted of 28 sounds chosen from the International Affective Digitized Sounds (IADS) archive (Stevenson \& James, 2008). For each sensory category, the stimuli were further extracted according to their arousal value. We selected 14 neutral faces and 14 fearful faces from the NIMSTIM inventory, and 14 low- and 14 high-arousal sounds were selected from the IADS repertoire and balanced by arousal with the NIMSTIM stimuli set. These materials are available here: https://doi. org/10.6084/m9.figshare.6874871.v3 (Tressoldi et al., 2018).

Experimental paradigm. All participants were presented with two different experimental tasks, which were delivered in separate blocks (see Figure 1). The first was a passive and the second was an active task preceded by a warm-up condition where the type of stimuli was anticipated in the cue signal. Both the block presentation order and the response button were counterbalanced between subjects to avoid possible response biases. The two tasks are described in the Figure 1.

Passive task. As shown in Figure 1, at the beginning of each trial, participants were presented with a warning signal, a fixation cross presented centrally on the screen for $300 \mathrm{~ms}$. After that, a fixed $1000 \mathrm{~ms}$ blank inter-stimulus interval (ISI) was delivered, followed by a 500ms target stimulus. The target stimulus could be either the picture of a face presented on the centre of the screen or a sound delivered bilaterally through two loudspeakers, with a $50 \%$ distribution. Half of the stimuli within each category were low-arousal and the other half were high-arousal, equally distributed. Participants were told that they had to guess which kind of stimulus they would be presented with. No behavioural responses were required until they actually received the stimulus target. At target onset, participants had to discriminate between visual or auditory stimuli by pressing two different buttons on the response box. The response buttons were counterbalanced across participants. After the response, the stimulus target disappeared and a blank screen was presented for a jittered duration between 1000 and $1200 \mathrm{~ms}$ (inter-trial interval) before the beginning of the next trial.

Active task. In the active task, event sequence and timing were the same as those in the passive task. As illustrated in Figure 1, the only difference in comparison with the passive task was that, after the prestimulus ISI, participants were presented with a slide showing a central question mark. They were then asked to make an explicit choice about the sensory category of the upcoming stimulus by pressing the response box. This allowed us to obtain an overt behavioural measure of the anticipation of random events as the percentage of correct responses compared to chance-level for each stimulus category. Immediately after participants' response, stimuli were presented for $500 \mathrm{~ms}$. As with the passive task, the response buttons were counterbalanced across participants.

A total of 200 trials sorted by stimulus category and arousal was presented in each task, for an experiment duration of about 18 minutes. In both tasks, stimuli presentation was fully randomized. Specifically, the trial-type randomization was generated online during the ISI by using a true random number generator (TrueRNG-2 ${ }^{\mathrm{TM}}$ ). The TrueRNG hardware uses the avalanche effect in a semiconductor junction to generate true random numbers. Randomization via an external TrueRNG device does not rely on seed-based randomization algorithms, but on current fluctuations within the device, assuring a true random distribution. The RNG was interfaced with the stimuli presentation software E-Prime ${ }^{\mathrm{TM}}$ 2.0.8.90.

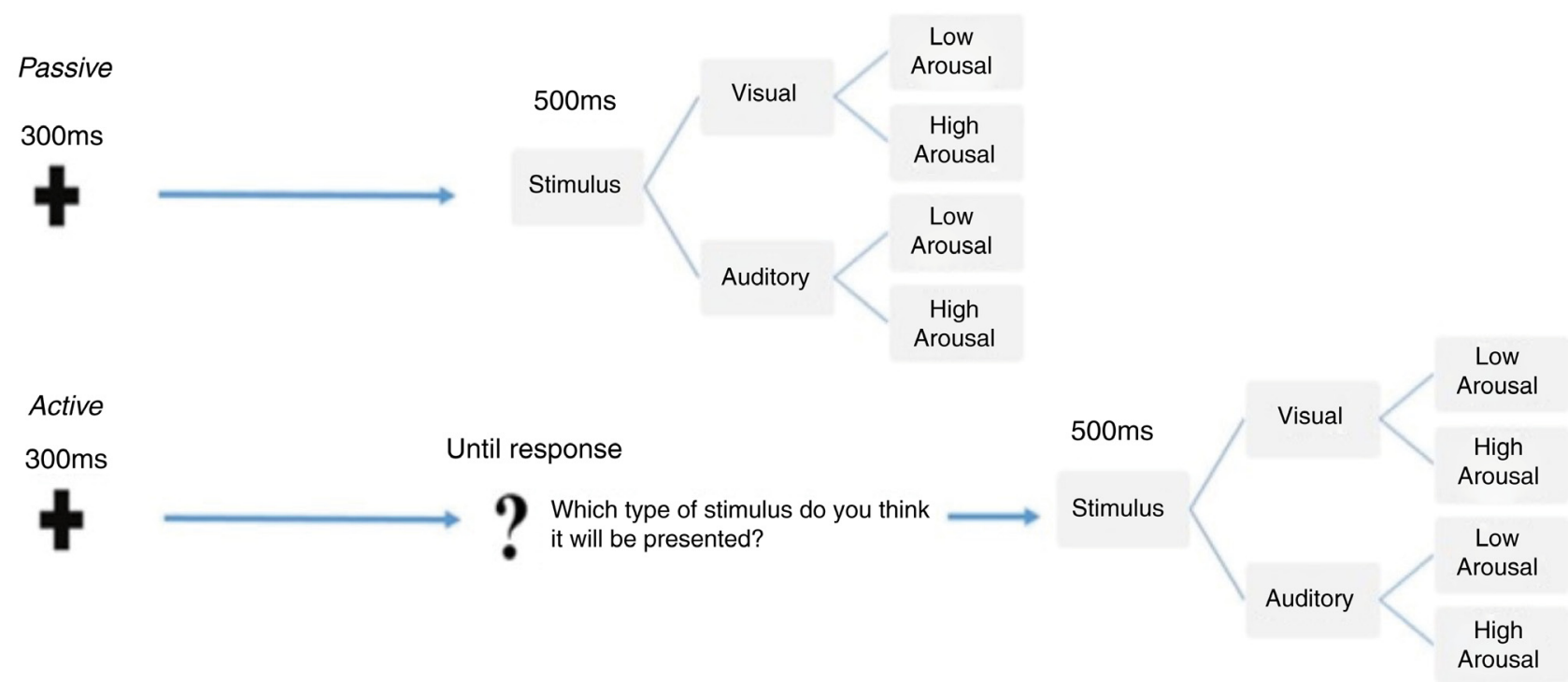

Figure 1. Experimental tasks. The figure illustrates the sequence of events and the temporal trial structure relative to the passive (top) and the active (bottom) tasks, which were delivered in blocks. Within each task, the stimuli were randomly presented and equally distributed according to either sensory category (faces or sounds) and arousal level (high or low). 


\section{EEG recordings}

During the entire experiment, the EEG signal was continuously recorded using a Geodesic high-density EEG system (EGI GES-300) through a pre-cabled 128-channel HydroCel Geodesic Sensor Net (HCGSN-128) and referenced to the vertex. Scalp voltages were recorded and amplified during the entire experiment. The sampling rate was $500 \mathrm{~Hz}$. The impedance was kept below $60 \mathrm{k} \Omega$ for each sensor. To reduce the presence of EOG artefacts, subjects were instructed to limit both eye blinks and eye movements as much as possible.

\section{Pre-registered data analysis}

Further detail about analysis, including removal of artefacts, is available from the preregistration record: https://osf.io/uf59a.

Event-related potential (ERP) analysis. The ERP analysis was performed with Matlab toolbox EEGLAB (Delorme \& Makeig, 2004). The EEG was segmented offline starting from $200 \mathrm{~ms}$ before the cue onset and ending $300 \mathrm{~ms}$ after the stimulus onset. The length of the analysed epoch was $1600 \mathrm{~ms}$, starting from cue onset and included $300 \mathrm{~ms}$ of cue/fixation cross presentation, $1000 \mathrm{~ms}$ of ISI, and $300 \mathrm{~ms}$ from stimulus onset. Our original hypothesis, driven by the results of our previous study (Duma et al., 2017), was that PPA phenomena may rely on a prestimulus sustained ERP activity, presumably resulting in the amplitude modulation of the CNV component. For this reason, our confirmatory, pre-planned analysis purposely targeted the temporal window between $300 \mathrm{~ms}$ and $1300 \mathrm{~ms}$ from trial onset.

All epochs were visually inspected to remove bad channels and rare artefacts. Artefact-reduced data were then subjected to Independent Component Analysis (Stone, 2002). All independent components were visually inspected, and those related to eye blinks, eye movements, and muscle artefacts according to their morphology and scalp distribution were discarded. The remaining components were then projected back to the electrode space to obtain cleaner EEG epochs. The remaining epochs containing excessive noise or drift $( \pm 100 \mu \mathrm{V}$ at any electrode) were rejected. After this step, removed bad channels were reconstructed. Data were then re-referenced to the average of all electrodes, and the signal was aligned to the baseline by subtracting the mean signal amplitude in the pre-stimulus interval. Subject average and grand average ERPs were generated for each electrode site and experimental condition. To provide a spatial representation of the anticipatory ERP activity, we used the Brainstorm software's dedicated software (Tadel et al., 2011). This allowed us to extract videos of obtained time-resolved scalp map projections of the grand average ERP activity from $0 \mathrm{~ms}$ to $1350 \mathrm{~ms}$.

Brain source analysis. Baseline corrected epochs (-200 to $0 \mathrm{~ms}$ ) were imported to Brainstorm software (Tadel et al., 2011) with the purpose of reconstructing the cortical generators of pre-stimulus ERP activity. Conductive head volume was modelled according to the Boundary Element Method (BEM) using Open MEEG toolbox (Gramfort et al., 2010; Kybic et al., 2005). The solution space was constrained to the cerebral cortex, which was modelled as a three-dimensional grid of 15002 vertices. Furthermore, the inverse transformation was applied to the Montreal Neurological Institute (MNI) canonical mesh (http://brainmap.org/training/BrettTransform.html) of the cortex to approximate real anatomy. The EEG sensor positions were co-registered with the default anatomical mesh by employing rigid rotations and translations of digitized landmarks (anterior and posterior commissure, interhemispheric scissure, nasion, and left and right tragus). The inverse modelling was based on sLORETA implemented as a routine of the Brainstorm platform. A noise covariance matrix was generated for each participant based on the average baseline time window. For each participant, the sources were projected using the standard anatomical template provided by the MNI, and their activity was transformed in $\mathrm{Z}$ scores relative to the baseline.

ERP statistical analysis. We compared face vs. sound prestimulus ERP activity $(300-1300 \mathrm{~ms})$ at the sensor level, including all 128 electrodes. Moreover, to further address the role of arousal in anticipation of random events, we also compared the high- and low-arousal stimuli within each sensory category. Given that a 128-channel recording system and a time resolution of $2 \mathrm{~ms}(500 \mathrm{~Hz})$ were employed, performing a systematic comparison of all samples present a severe risk of false positives. Hence, we used a non-parametric, mass-univariate statistical approach based on paired t-test permutations (Groppe et al., 2011) already employed by our group in previous ERP studies (Duma et al., 2017; Mento et al., 2018; Mento, 2017; Mento \& Vallesi, 2016; Mento \& Valenza, 2016). Specifically, 1000 Monte Carlo permutations with cluster correction were applied, using Fieldtrip (Oostenveld et al., 2011) functions, implemented in Brainstorm.

Exploratory analysis. The above-mentioned statistical analyses were preregistered and hypothesis driven. We expected to find that the presence of anticipatory effects of random stimuli was reflected in an amplitude change of the CNV component elicited during the ISI. However, it should be noted that the real nature of anticipatory brain activity of statistically unpredictable stimuli has not been fully explored. Hence, it is possible that the exact spatial and temporal locus of PPA effects could be outside the time windows expected based on previous evidence. Thus, while the use of a confirmatory approach increases the reliability and reproducibility of the results, it may exclude possible interesting effects extending over temporal windows or spatial areas originally ignored in the pre-declared analyses. For this reason, we implemented additional exploratory analyses extending the epoch of interest to the entire pre-stimulus time window (i.e., from the onset of the fixation cross to the end of stimulus presentation) with the aim of looking for possible earlier effects than those expected. To test for any data-driven difference between experimental conditions, a Support Vector Machine (SVM) analysis was performed. The SVM belongs to the class of supervised machine learning models. Given a set of training data, in which the two categories are labelled, the SVM algorithm generates a model that is able to assign new examples to one category or the other. The 
K-folds cross-validation technique implemented in Brainstorm (Tadel et al., 2011) has been used to validate the SVM model using the ERP of each subject as data. Crossvalidation is a resampling procedure for model selection. K-fold approach 'involves randomly dividing the set of observations into $\mathrm{k}$ groups, or folds, of approximately equal size. The first fold is treated as a validation set, and the method is fit on the remaining $\mathrm{k}-1$ folds' (James et al., 2013). For each task, we contrasted face and sound stimuli and high- and low-arousal stimuli separately within each stimulus category. The results of the SVM approach are expressed as the dynamic, time-resolved evolution of the discrimination accuracy between two categories. It worth mentioning that Machine Learning (ML) approach with EEG signal shows different results based one the features of the EEG signal to classify, the feature selection criterion and the classifier algorithm (Lotte et al., 2007). Moreover, to the best of our knowledge, ML approach have never been applied to classify brain activity for statistical unpredictable stimulus presentation. For these reasons, we tested different cut-off frequency $(20 \mathrm{~Hz}, 10 \mathrm{~Hz}, 7 \mathrm{~Hz}, 5 \mathrm{~Hz}, 4 \mathrm{~Hz}$, and $3 \mathrm{~Hz})$ in order to understand which frequency maximized the classification accuracy of the algorithm.

\section{Results}

\section{Behavioural findings}

The descriptive statistics relative to the percentages of the correct predictions of faces and sounds in the active block are reported in Table 1.

\section{Sensor-level ERP}

The mean number of artefact-free trials per subject accepted for averaging was comparable both in the passive (Faces: $90 \pm 7.79$; Sounds: $92 \pm 9.3$ ) as well as in the active (Faces: $90.60 \pm 10.1$; Sounds: $99 \pm 10$ ) task. Likewise, the number of high- and low-arousal stimuli within each stimulus category was comparable both in the passive (Low Arousal Faces: $46.6 \pm 6.34$; High Arousal Faces: $43.4 \pm$ 4.52; Low Arousal Sounds: $46.5 \pm 6.6$; High Arousal Sounds: $45.67 \pm 6.83$ ) and

active task (Low Arousal Faces: $46.21 \pm 7.6$; High Arousal Faces: $44.4 \pm 6.8$, Low Arousal Sounds: $45.35 \pm 7.57$; High Arousal Sounds: $43.68 \pm 6.56$ ). This confirmed that the true-random trial sorting procedure was effective in generating an equivalent number of trials per condition, ensuring a comparable ERP signal-to-noise ratio.

As expected based on previous literature (Mento et al., 2013; Walter et al., 1964), the visual inspection of ERP waveforms revealed the presence of a reliable CNV component in all tasks. This component was represented as an increase in frontocentral negativity about $500 \mathrm{~ms}$ from trial onset and lasting for the full duration of the ISI duration until stimulus onset. The topographical distribution of this activity for each task is shown in Figure 2 and Figure 3 for face vs. sound and high- vs. low-arousal contrasts, respectively (see also https://doi. org/10.6084/m9.figshare.6874871.v3 (Tressoldi et al., 2018) for a time-resolved dynamic spatial representation of the CNV over the whole scalp).

\section{Faces vs. sounds}

The visual inspection of the high scalp-resolution maps obtained by averaging the $\mathrm{CNV}$ time window (i.e., 300-1300ms) showed prestimulus differences in the topographical distribution between faces and sounds, with a greater fronto-central negativity for face type stimuli (Figure 2). This difference was less evident in the active block.

High- vs. low-arousal stimuli

Concerning arousal level, the scalp distribution maps displayed an increase in the fronto-central negativity for high-arousal face stimuli compared to low-arousal stimuli in the passive task, as well as a differential map localization of the CNV between high- and low-arousal sound stimuli. Notably, this brain activity pattern was not consistent across tasks. In the active task, low- and high- arousal stimuli elicited a comparable frontocentral negativity, although a larger posterior positivity was evident for low-arousal face and high-arousal sound (Figure 3).

Although the visual inspection of the spatial maps revealed qualitative topographical differences, in none of the two tasks

Table 1. Means and
standard deviations
of the percentages
of correct faces and
sounds predictions.

\begin{tabular}{|l|l|l|}
\hline & Faces & Sounds \\
\hline Mean & 52.6 & 51.0 \\
\hline SD & 5.7 & 5.7 \\
\hline
\end{tabular}

The inferential statistics based on a one-tailed, one-sample t-test with the null hypothesis of $50 \%$ corresponding to the chance level yielded the following results: Faces, t-test = 2.3; $p=.01$; effect size $d=.45$; $95 \% \mathrm{Cl}=.019-.85$; Sounds $\mathrm{t}$-test $=.97 ; \mathrm{p}=.16 ;$ effect size $\mathrm{d}=.19 ; 95 \% \mathrm{Cl}=-.20-.60$

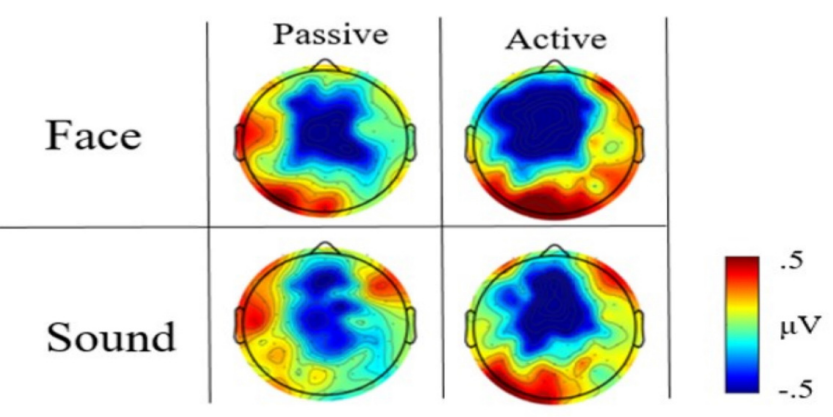

Figure 2. Scalp maps of the mean activity in the $300-1300 \mathrm{~ms}$ prestimulus time window in passive (central column) and active (right column) tasks for face (first row) and sound (second row) stimuli after collapsing the arousal level. 


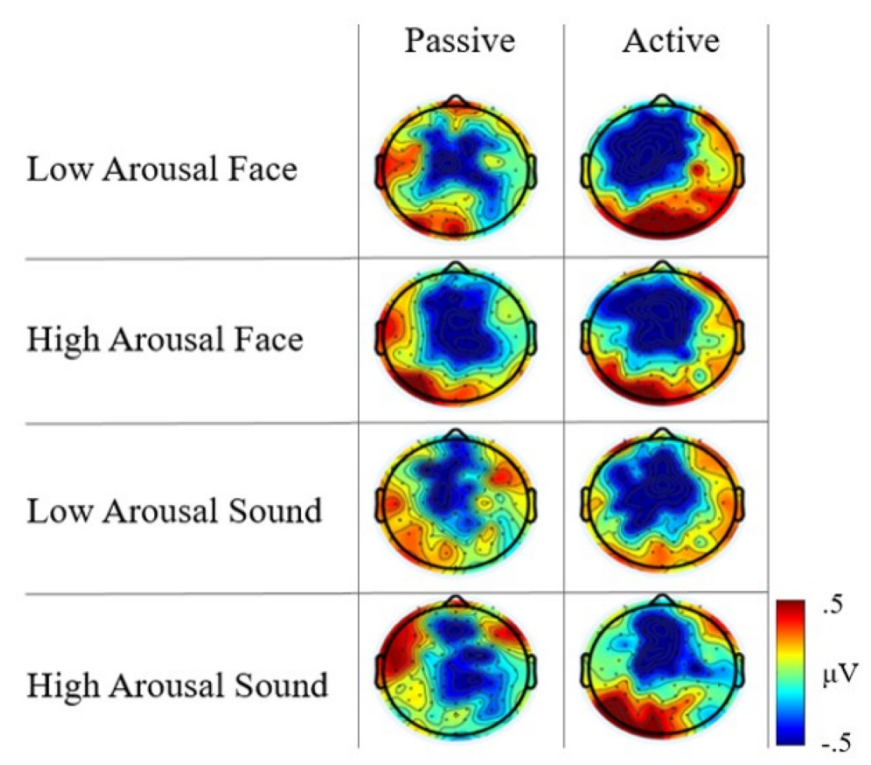

Figure 3 . Scalp map mean activity in the $300-1300$ ms prestimulus window in the passive (left column) and active (right column) task for faces and sound (high and low arousal, third and fourth row, respectively).

did the non-parametric, permutation analyses performed in the pre-registered anticipatory epoch $(300-1300 \mathrm{~ms}$ from warning onset) reveal statistically significant effects, whether when comparing stimulus categories or when contrasting stimuli arousal within categories.

A possible explanation of the lack of significant statistical corroboration of visual differences may be that mass univariate statistical tests are strongly sensitive to the presence of random factors, as in the case of including the whole spatial information (128 electrodes). This may ultimately result in excluding experimental effects strictly localized in specific scalp regions or temporal windows. As mentioned above, the hypotheses about the spatio-temporal properties of PPA induced by the present experimental manipulations were driven by our previous study (Duma et al., 2017), which showed a broad fronto-central and long lasting $\mathrm{CNV}$ modulation in anticipation of random simulated car accidents. However, in that study, we used a low spatial resolution EEG system (32 vs. 128 channels) as well as a lower signal acquisition sampling rate (i.e., 250 vs. $500 \mathrm{~Hz}$ ). In hindsight, this methodological difference may explain the disappearance of significant results due to a strong multiple-comparison correction. In line with this possible explanation, we observed that the fronto-central electrode activity in both tasks showed significant modulatory effects when permutations were performed without applying cluster-based correction for controlling Type 1 error.

\section{Source level results}

Our pre-registered analyses included the reconstruction of the source level maps. Although the massive cluster-based correction for multiple comparisons did not yield statistically significant observed differences between the conditions, we report the cortex activation results in Figure 4.

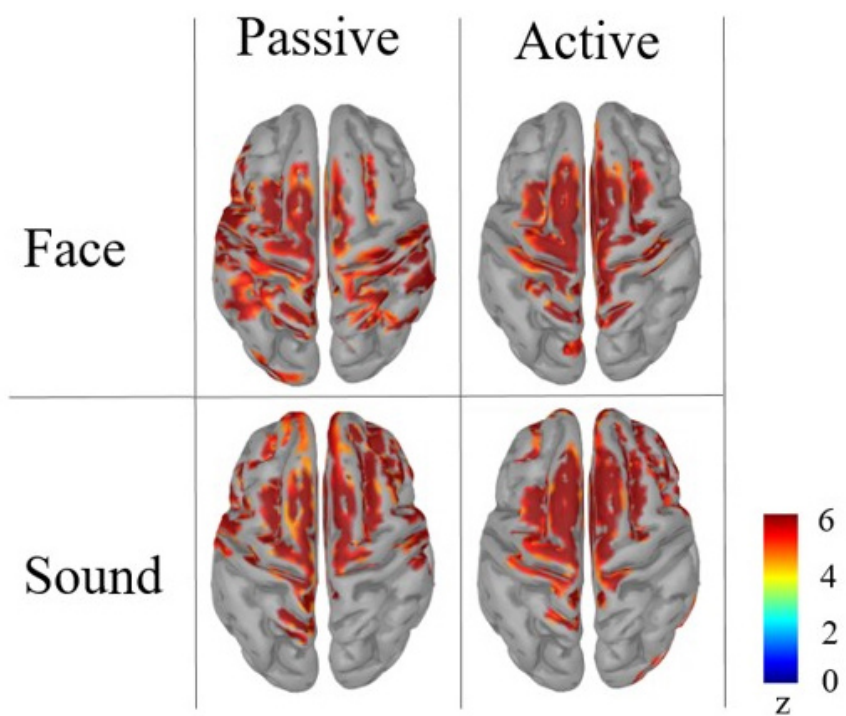

Figure 4. Normalized source maps of the mean activity in the pre-stimulus time window (between $300-1300 \mathrm{~ms}$ ) relatively to each task and condition.

From the cortical source map reconstruction, it is possible to identify a common activation for both tasks and stimulus categories in the superior frontal gyrus bilaterally, namely the Supplementary Motor Area that in literature has been identified as one of the most reliable neural generators of CNV (Mento et al., 2013; Mento et al., 2015; Mento, 2017). In addition to this, the cortical maps suggest the possible presence of stimulus-driven differences in primary sensory areas. In spite of this, the source-level results can be considered merely descriptive, rather than offering reliable hints for any inferential reasoning. In fact, considering the absence of statistically significant results at the sensor level, any strong interpretation from source space findings is to be discouraged as a precaution. While bearing these methodological issues in mind, one should nonetheless take into account that both sensor- and source-level descriptive results could be deemed suggestive of the possible presence of subtle effects to be further explored with ad-hoc analyses. To follow up on this indication, we performed additional exploratory statistical analyses extending over earlier temporal windows in an attempt to specifically focus on the possible presence of specific, fine-grained experimental effects.

\section{Exploratory results}

Support vector machine results. The data were analyzed with SVM algorithm by applying different exploratory frequency thresholds $(20 \mathrm{~Hz}, 10 \mathrm{~Hz}, 7 \mathrm{~Hz}, 5 \mathrm{~Hz}, 4 \mathrm{~Hz}$, and $3 \mathrm{~Hz}$, with a folding parameter of 5). The best algorithm performance in terms of experimental condition discrimination was obtained by applying a $4 \mathrm{~Hz}$ threshold, which was then set as the target SVM frequency. As shown in Figure 6, the application of SVM in the passive task succeeded in discriminating between Face and Sound in the time window $100-300 \mathrm{~ms}$, reaching a discrimination accuracy value of $71.43 \%$ (see Figure 5). 


\section{Face vs Sound stimuli}

\section{High vs Low Arousal Face stimuli}

\section{High vs Low Arousal Sound stimuli}
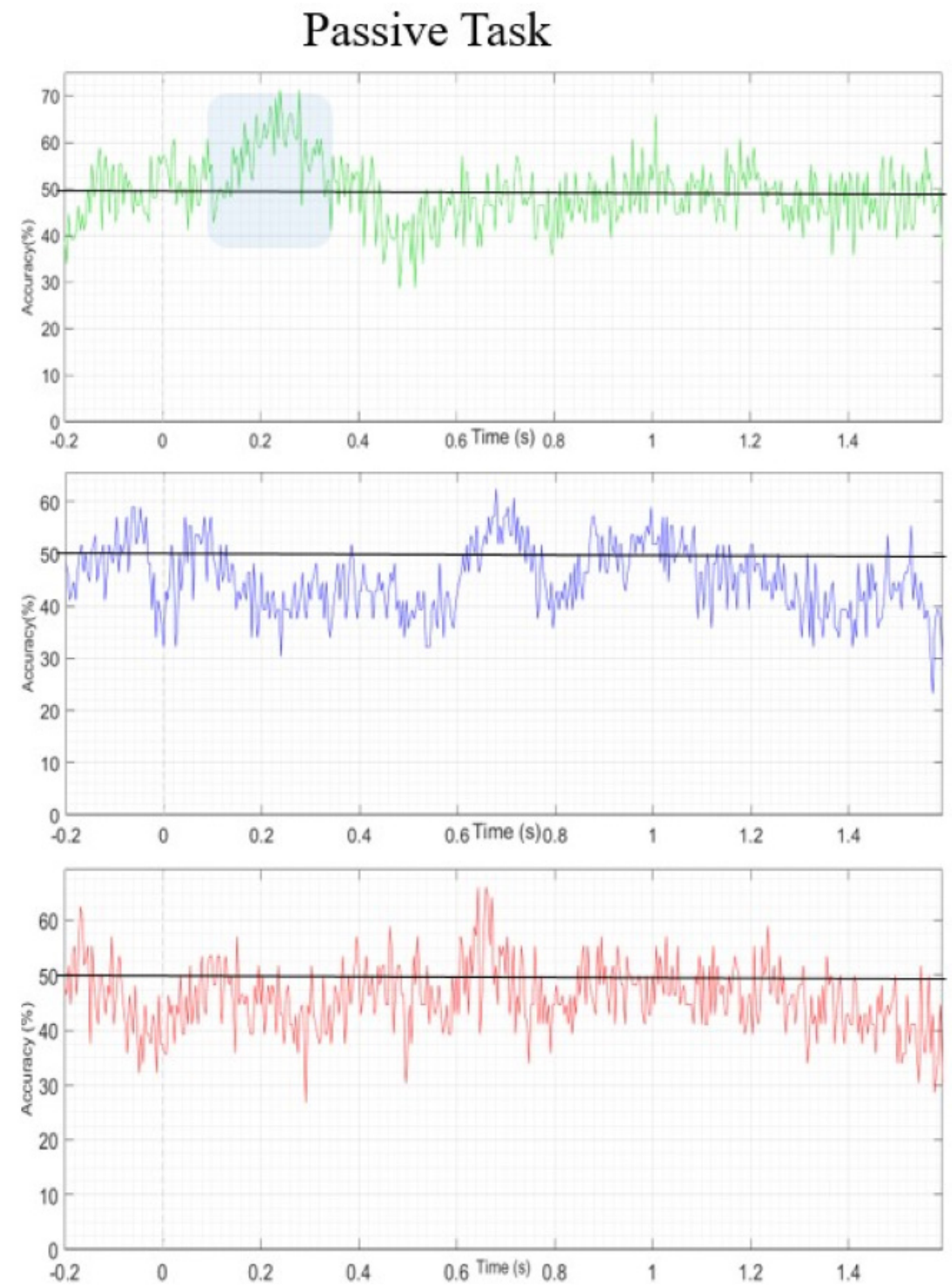

Figure 5. Support Vector Machine decoding accuracy in time in the passive task.

In the active task, the SVM was able to correctly differentiate between high- and low-arousal sounds, reaching a decoding accuracy of $68 \%$, but in a later temporal window, between 1100 and 1300ms (see Figure 6).

To statistically confirm the SVM findings while accounting for all spatial information, we applied cluster-based permutation statistics taking into account all 128 channels, this time targeting the specific contrasts and temporal windows indicated by the SVM algorithm as exhibiting effects.

Concerning the passive task, the results showed a bilateral posterior significant cluster of electrodes $(p=.01966$, cluster statistic $=39$, cluster size $=28$ ) when we compared face and sound stimuli in the $100-300 \mathrm{~ms}$ time window. Concerning the active task, we found a significant left centro-posterior cluster $(\mathrm{p}=.047$, cluster statistic $=25$, cluster size $=18)$ when higharousal sounds are compared to low-arousal sounds. These differences are presented in Figure 7.

SVM guided source level

To further address the functional nature of these phenomena, we performed the reconstruction of the cortical source of both effects described above. Both the source-level topological distribution (source mean activity between 300-1300ms) and temporal evolution over the whole prestimulus epoch are depicted in Figure 8.

Relative to the early anticipatory effect, we observed a higher recruitment of the occipital-parietal left cortical regions, 


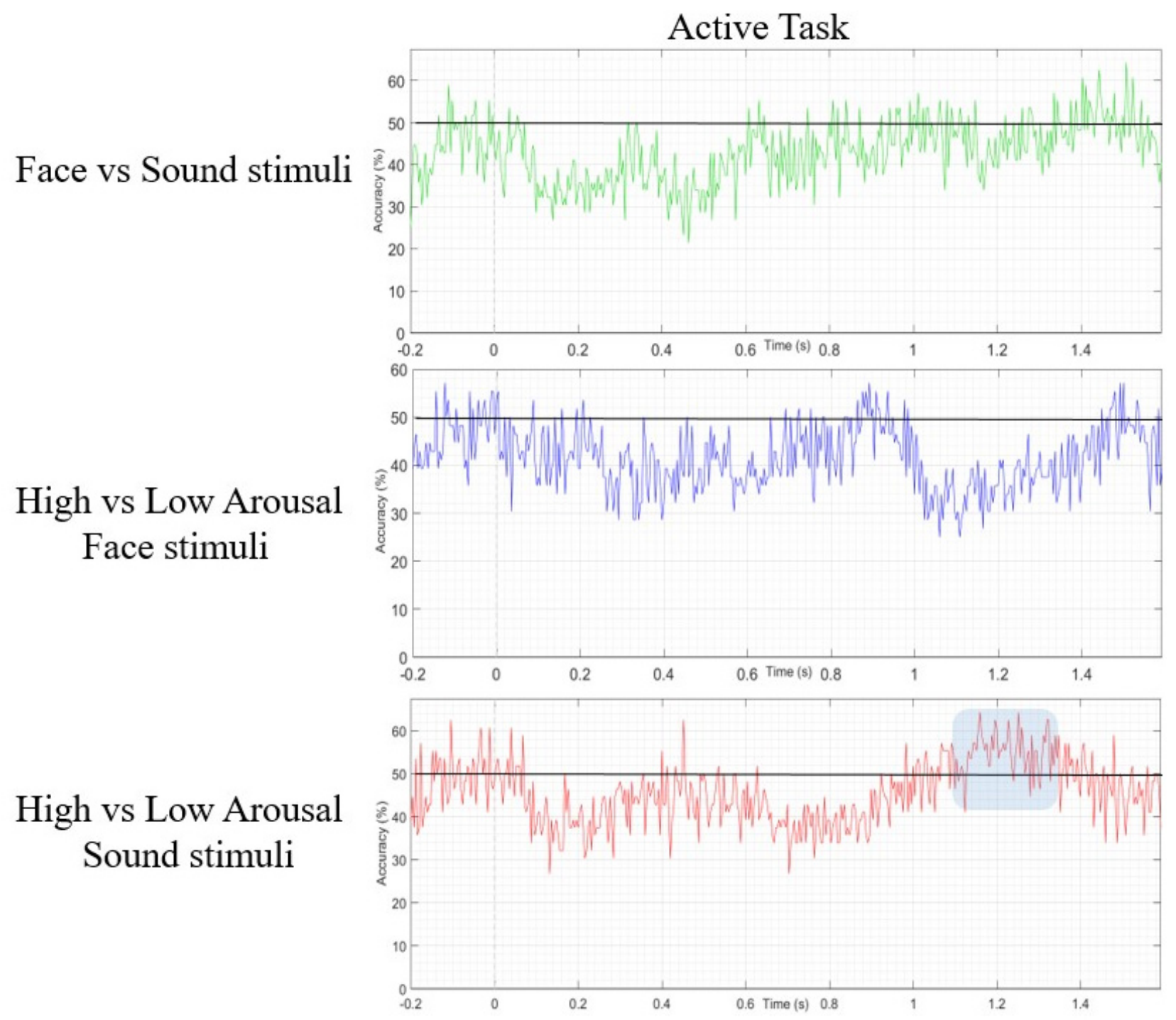

Figure 6. Support Vector Machine Decoding accuracy in time in the active task.

(A)

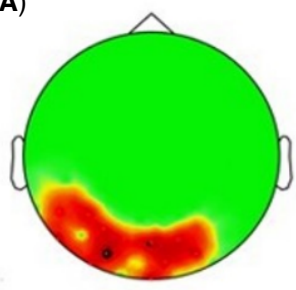

Face vs Sound Stimuli Passive Task $100300 \mathrm{~ms}$
(B)

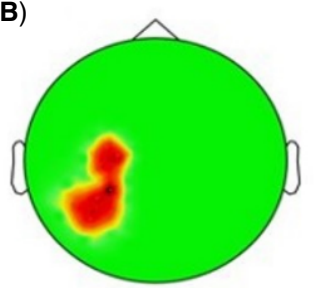

High vs Low arousal Stimuli Active Task $11001300 \mathrm{~ms}$

Figure 7. Statistically significant differences in the $\mathbf{1 0 0}-\mathbf{3 0 0 m s}$ time window. a) in the occipital cluster for face vs sound stimuli comparison in the passive task; b) in the parietal cluster for high- vs low-arousal sound comparison in the active task. 

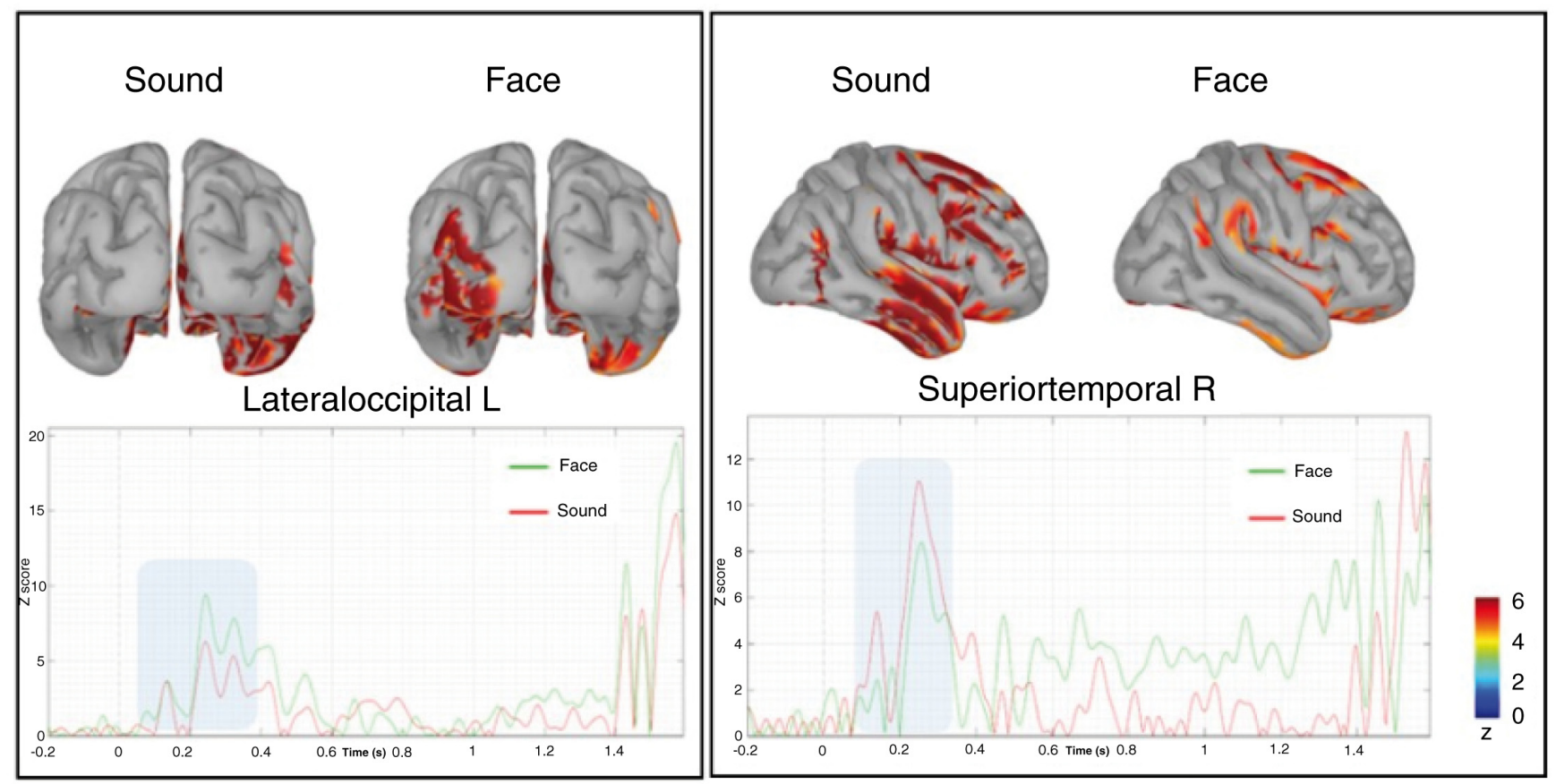

Figure 8. The source activations represent the mean activity in the source space between 100-300ms (neutral fixation cross time window). The time series below represent the time evolution of the source activation, highlighting the difference between 100-300ms (blue rectangle), respectively of the lateral occipital left (on the left) and superior temporal right (on the right).

especially the lateral occipital gyrus, which was more activated before the presentation of faces regardless of the arousal they expressed. The visual inspection of the temporal dynamics showed that this region is highly activated at about $200 \mathrm{~ms}$ from the presentation of the fixation cross when a face was presented. No other relevant modulations are present in the late prestimulus interval spanning the $\mathrm{CNV}$ timing. A second remarkable finding was a larger recruitment of neural activity in the right superior temporal gyrus, again at about $200 \mathrm{~ms}$ when a sound was presented. Both of the above findings corroborate our original, pre-declared hypothesis that PPA brain activity is category-dependent, since it engages in the same primary sensory areas that will be activated after target presentation. In other words, the anticipation of a face preactivates visual occipital areas lefts lateralized, whereas the anticipation of a sound pre-activates auditory temporal areas more lateralized in the right hemisphere.

When performed on the second temporal window showing late prestimulus ERP amplitude modulation between high- and low-arousal auditory stimuli in the active task, the cortical reconstruction showed that high-arousal stimuli were preceded by the recruitment of a broader cortical network as compared to low-arousal stimuli. Indeed, in both cases, the fronto-central areas around the SMA were commonly activated. However, when the fixation was actually followed by the presentation of high-arousal stimuli, we observed an additional involvement of the left superior parietal area, maximally expressed between 1100 and 1300ms from its onset (see Figure 9).

\section{Discussion}

In a recent study (Duma et al., 2017), we reported evidence of differential anticipatory brain activity preceding unpredictable, simulated car crashes or safe journeys. Here, we sought to further investigate the evidence of psychophysiological predictive activity (PPA; Mossbridge et al., 2014) of random events while further ruling out potential methodological shortcomings due to the randomization process and stimulus timing. As a general consideration, it must be taken into account that the available evidence in existing literature about the neurophysiological mechanisms underpinning the anticipation of random events is limited. Hence, it is difficult to generate clear and reliable hypotheses with regard to the spatial and temporal neural locus of such phenomena. Notwithstanding this caveat, a commonly accepted idea is that if the brain is able to anticipate statistically unpredictable random stimuli, different forthcoming stimuli categories should rely on specific and dissociable anticipatory neural patterns. More precisely, this anticipatory effect might be reflected in the pre-activation of the brain regions that subtend the elaboration of the physical features of upcoming stimuli (e.g., a pre-activation of the auditory cortical regions preceding the onset of a sound as well as of visual regions preceding face presentation). Following this line of reasoning in our previous work, we had argued that the 


\section{Sound Stimuli}

High Arousal

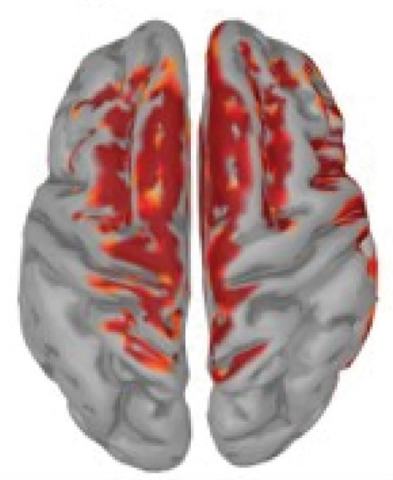

Low Arousal

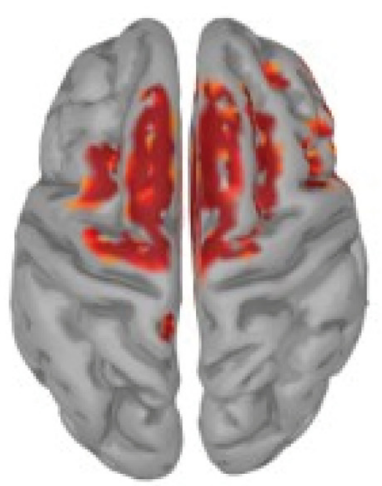

\section{Superiorparietal L}

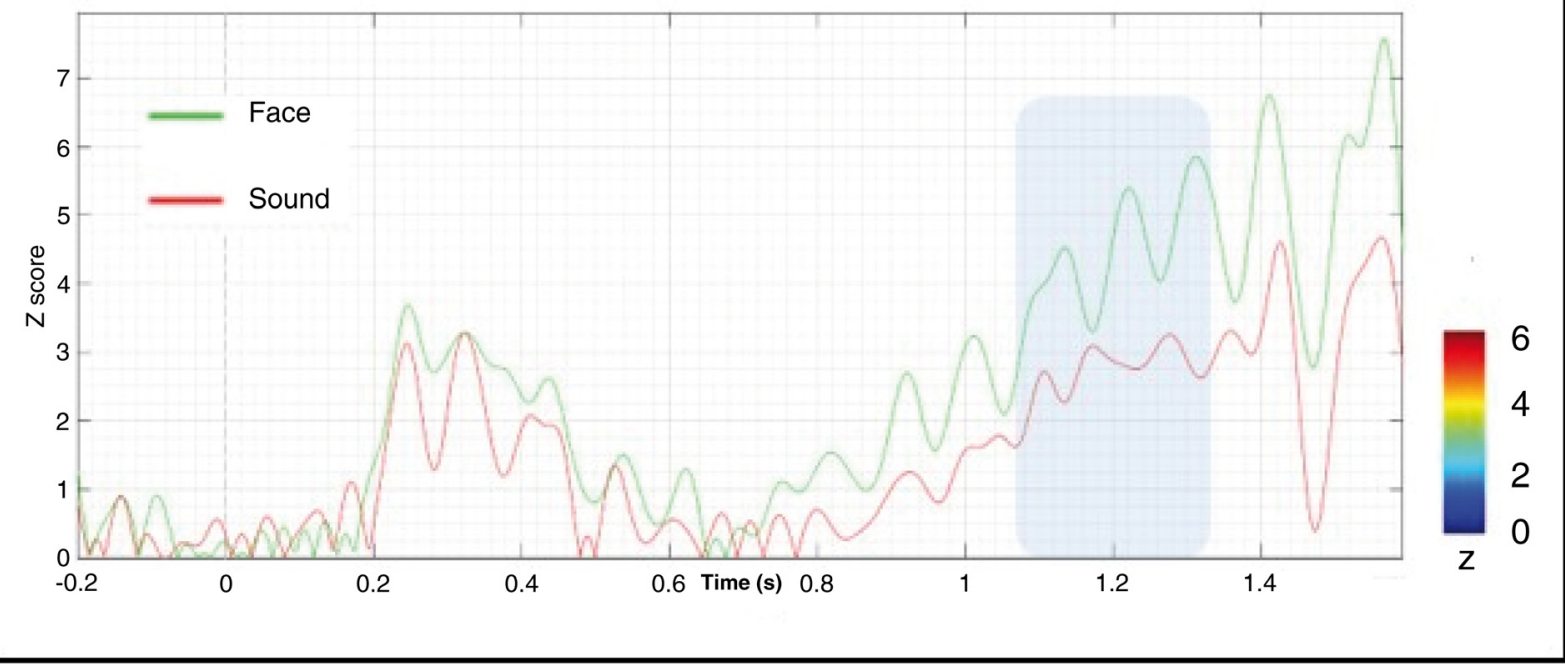

Figure 9. Mean activity in 1100-1300ms time window of high- and low-arousal sound stimuli in the active block. Below in the figure, it is represented the time course of the superior parietal left area of the Desikan-Killaney atlas.

capability of anticipating potentially threatening events (e.g. simulated car accidents) may result in the mobilization of psychophysical resources to implement a possible reaction, this mechanism putatively playing a key role in survival.

Based on the above assumptions, the aim of the present study was two-fold. On the one hand, we tried to address some potential methodological issues that may have somehow biased our previous study. These included either the implementation of a pseudo-random instead of truly random trial sorting procedure or the use of a variable temporal trial structure. On the other hand, we tried to extend our previous results into other stimuli category with the aim of deepening the understanding of the sources of anticipatory brain activity of randomly presented stimuli. 
To address these goals in the present study, we recorded the high spatial resolution EEG activity when presenting two different stimulus categories in a random, unpredictable sequential order. These included pictures of faces and sounds as visual and auditory sensory categories, respectively. Moreover, we further manipulated the psychophysiological activation meaning of those categories by matching them by arousal level (high or low). All stimuli were randomly presented by interfacing with a true random number generator. As in our original hypothesis, we expected to identify at the EEG sensor level a stimulus type and/or arousal level dependent difference during the prestimulus time window. Specifically, following our pre-registered hypothesis we expected to observe some stimulus-driven amplitude differential effects arising from about $1000 \mathrm{~ms}$ before stimulus onset. Moreover, we hypothesized that such differences may stem from specific neural patterns mimicking the sensory nature of the stimulus. That is, we expected to observe source-level pre-activation of visual cortical areas before faces and pre-activation of auditory cortical areas before sounds.

The cluster-based permutation analyses of the target prestimulus time window (i.e., $300-1300 \mathrm{~ms}$ from trial onset) showed only a marginally significant $\mathrm{CNV}$ modulation due to experimental manipulation. Indeed, the application of a massive cluster-based multiple-comparison correction taking into account the whole spatial information (128 electrodes) as well as a huge temporal window (as long as 1 second, i.e., between 300 and $1300 \mathrm{~ms}$ from trial onset) did not confirm this effect from a statistically point of view.

As mentioned above, a possible explanation for this null effect is that the implementation of a whole-scalp analysis may have been too conservative for a subtle experimental effects. In fact, in our previous study (Duma et al., 2017) we used a lower resolution EEG system (32 electrodes), which ultimately resulted in a considerably smaller number of multiple comparisons. In other words, the analysis of a large, complex spatiotemporal dataset may have requested a stronger statistical power.

However, we decided to further analyse our data by applying an exploratory approach. To this purpose we used an SVM as a machine-learning classificatory algorithm. In the passive task, this analysis revealed a data-driven discrimination accuracy of $71.43 \%$ between visual and auditory stimuli which occurred in an early temporal window (100-300ms from trial onset). In the active task, the SVM algorithm revealed a later data-driven effect (maximum peak 68\%) spanning in the late $\mathrm{CNV}$ range (1100-1300ms from trial onset), which was specific for the arousal level. It should be underlined that SVM results have been used as a guide indicating in which conditions and temporal windows focusing the analysis. These results were further confirmed by a cluster-based permutation statistics, which were performed targeting the specific temporal windows identified by the SVM. In fact, by applying cluster-based permutation statistics over the temporal window (100-300ms) and conditions (passive face vs. sound), as evidenced by SVM results, we observed a significant bilateral occipital cluster of electrodes showing larger amplitude when the fixation cross at trial onset was followed by visual than auditory stimuli. These prestimulus activities were supported by different cortical networks. Indeed, we found that the visual areas (i.e., the left lateral occipital lobe) were more activated at about $200 \mathrm{~ms}$ from trial onset in trials displaying faces than in those delivering sounds. Differently the right auditory cortex was more activated in trials containing sounds than in those delivering faces.

Additionally, we found that in the active task when participants were explicitly asked to guess the category of the forthcoming stimulus (face or sound) the percentage of correct responses $(52 \%)$ was significantly higher than chance level. This behavioural finding was accompanied by a larger CNV activity in the late prestimulus interval (1100-1300ms) for high- vs. low-arousal stimuli. This effect was localized in the left centro-posterior scalp regions and originated from a specific pre-activation of the left superior parietal cortex, which showed a larger sustained activity before high-arousal stimuli in the late prestimulus interval. Although the exact functional role of the superior parietal cortex in the anticipation of random, high-arousal sounds is not well understood on the basis of current scanty literature, a possible generic explanation may maintain that this region is involved in the pre-allocation of cognitive resources needed to process predicted high-arousal stimuli.

Although obtained by exploratory, rather than originally planned, confirmatory hypotheses, these results are in line with the expectations of a stimulus-dependent pre-activation of those brain regions that will be engaged in processing the upcoming stimulus.

While we feel reasonably confident in excluding that the presence of anticipatory effects of random events may have been biased by possible methodological shortcomings, the interpretation of the possible nature of such phenomena is still debated. In line with one of the most shared theoretical accounts, our results may be explained in the theoretical framework of the Predictive Anticipatory Activity (PAA) of Mossbridge et al. (2014). The underlying assumption of this kind of empirical evidence maintains that PAA might be thought of as an unconscious anticipation of a conscious future events, suggesting the possibility of quantum-like temporal entanglement effects in human physiological processes (Fisher, 2015; Hameroff, 2012; Jedlicka, 2017; Tressoldi et al., 2015). The endorsement of quantum-like phenomena as possible mediating mechanisms of physiological processes taking place in random event prediction, implies the possibility of temporal symmetry in the interpretation of reality (Reznik \& Aharonov, 1995) or even possible retrocausal effects (Ma et al., 2012).

Whatever the specific physical and physiological nature of anticipatory mechanisms, which is beyond the original purpose of the present study, we report a methodologically-controlled and replicable experimental setting that suggests the reliability of PPA phenomena. To the best of our knowledge, this is the first work in which advanced techniques, such as 
high-spatial resolution EEG and source reconstruction, have been applied to the investigation of PPA phenomena in combination with the implementation of both confirmatory and exploratory methodological approaches. This allowed us to present new data complementing previous evidence because we argue that PPA phenomena may be driven by specific brain processes, which involves discrete brain areas according to the sensorial category of the upcoming stimulus.

In scientific literature, criticism have been raised regarding the anticipatory effects for statistically unpredictable stimuli (Galak et al., 2012; Ritchie et al., 2012; Wagenmakers et al., 2011), criticizing the findings reported by Bem (2011). Nonetheless, we believe that those criticisms cannot be related to our work, both for theoretical and methodological reasons. First, Bem's results were referred to the so called 'Retroactive Facilitation of Recall'. In fact, as stated by the author, in his experimental task "Participants were first shown a set of words and given a free recall test of those words. They were then given a set of practice exercises on a randomly selected subset of those words. The psi hypothesis was that the practice exercises would retroactively facilitate the recall of those words, and, hence, participants would recall more of the to-bepracticed words than the unpracticed words" (Bem, 2011). By contrast, our theoretical framework emerges from the already published results of Radin et al. (2011), Mossbridge et al. (2012), Mossbridge et al. (2014), and Duma et al., 2017, who investigated physiological activity (EEG, EMG, pupillary response) before stimuli randomly presented, finding positive results. Therefore, considering the evidence already published, and the evident difference between Bem's study and the one we are presenting, both in the type of measures (behavioural vs. electrophysiological), and the experimental paradigm (words recall vs. face/sound passive presentation), we believe that the possible criticisms raised by the abovementioned authors cannot be by default applied to all the studies investigating anticipatory phenomena, including the present one.

We are aware that the present topic needs additional confirms from scientific literature. For this reason, we believe that the present results may be a stimulus to other researchers to investigate, with a rigorous and fully reproducible methodological approach, the real nature of anticipatory effects for statistically unpredictable stimuli, deepening in this way our understanding.
For example, future replications of the present study may include single-subject MRI scans with the objective of localizing a single subject's visual and auditory cortex, using those areas as ROI to be entered in a multivariate statistic approach. Taking in account the growing body of literature providing experimental results to the human capability of anticipating randomly presented stimuli, we believe that this topic deserves a precise and rigorous scientific investigation, as it happens for all cognitive human activities, in order to understand this controversial but nevertheless fascinating phenomena.

\section{Data availability}

Underlying data

Figshare: EEG anticipation of random high and low arousal faces and sounds, https://doi.org/10.6084/m9.figshare.6874871.v4 (Tressoldi et al., 2018).

This project contains that following underlying data:

- EEG metafile;

- EEG data related to the Passive, Active and Predictive conditions;

- High (H) and Low (L) arousal visual and auditory stimuli;

- Video clips of the EEG activity before stimulus presentation.

Data are available under the terms of the Creative Commons Attribution 4.0 International license (CC-BY 4.0).

NIMSTIM archive materials available from http://www.macbrain.org/resources.htm. Registration and acceptance of the terms and conditions of the website must be completed in order to use the stimulus set.

International Affective Digitized Sounds (IADS) archive materials available from: https://csea.phhp.ufl.edu/media/iadsmessage.html. Those wishing to use these materials must submit the IADS Researcher Information Form and sign the IADS User Agreement.

\section{Grant information}

The author(s) declared that no grants were involved in supporting this work.
Bem DJ: Feeling the future: experimental evidence for anomalous retroactive influences on cognition and affect. J Pers Soc Psychol. 2011; 100(3): 407-425. PubMed Abstract | Publisher Full Text

Chater N, Tenenbaum JB, Yuille A: Probabilistic models of cognition: conceptual foundations. Trends Cogn Sci. 2006; 10(7): 287-291. PubMed Abstract | Publisher Full Text

Delorme A, Makeig S: EEGLAB: an open source toolbox for analysis of single-trial EEG dynamics including independent component analysis. J Neurosci Methods.
2004; 134(1): 9-21.

PubMed Abstract | Publisher Full Text

Duggan M, Tressoldi P: Predictive physiological anticipatory activity preceding seemingly unpredictable stimuli: An update of Mossbridge et al's metaanalysis [version 2; peer review: 2 approved]. F1000Res. 2018; 7: 407. PubMed Abstract | Publisher Full Text | Free Full Text Duma GM, Mento G, Manari T, et al.: Driving with Intuition: A Preregistered Study about the EEG Anticipation of Simulated Random Car Accidents. PLOS 
One. 2017; 12(1): e0170370

PubMed Abstract | Publisher Full Text | Free Full Text

Fisher MP: Quantum cognition: the possibility of processing with nuclear spins in the brain. Ann Phys. 2015; 362: 593-602.

Publisher Full Text

Friston K: A theory of cortical responses. Philos Trans $R$ Soc Lond B Biol Sci. 2005; 360(1456): 815-836.

PubMed Abstract | Publisher Full Text | Free Full Text

Galak J, LeBoeuf RA, Nelson LD, et al:: Correcting the past: failures to replicate \%. J Pers Soc Psychol. 2012; 103(6): 933-948.

PubMed Abstract | Publisher Full Text

Gramfort A, Papadopoulo T, Olivi E, et al.: OpenMEEG: opensource software for quasistatic bioelectromagnetics. Biomed Eng Online. 2010; 9(1): 45

PubMed Abstract | Publisher Full Text | Free Full Text

Groppe DM, Urbach TP, Kutas M: Mass univariate analysis of event-related brain potentials/fields I: a critical tutorial review. Psychophysiology. 2011; 48(12): 1711-1725.

PubMed Abstract | Publisher Full Text | Free Full Text

Hameroff S: How quantum brain biology can rescue conscious free will. Front Integr Neurosci. 2012; 6: 93

PubMed Abstract | Publisher Full Text | Free Full Text

James G, Witten D, Hastie T, et al:: An introduction to statistical learning. New York: springer. 2013; 112: 181

Publisher Full Text

Jedlicka P: Revisiting the Quantum Brain Hypothesis: Toward Quantum (Neuro)biology? Front Mol Neurosci. 2017; 10: 366

PubMed Abstract | Publisher Full Text | Free Full Text

Kybic J, Clerc M, Faugeras O, et al:: Fast multipole acceleration of the MEG/EEG boundary element method. Phys Med Biol. 2005; 50(19): 4695-710. PubMed Abstract | Publisher Full Text

Lotte $\mathrm{F}$, Congedo M, Lécuyer $\mathrm{A}$, et al: : A review of classification algorithms for

EEG-based brain-computer interfaces. J Neural Eng. 2007; 4(2): R1-R13.

PubMed Abstract | Publisher Full Text

Ma XS, Zotter S, Kofler J, et al.: Experimental delayed-choice entanglement swapping. Nat Phys. 2012; 8(6): 479-484.

Publisher Full Text

Mento G: The role of the P3 and CNV components in voluntary and automatic temporal orienting: A high spatial-resolution ERP study. Neuropsychologia. 2017; 107: 31-40.

PubMed Abstract | Publisher Full Tex

Mento G, Astle DE, Scerif G: Cross-frequency Phase-Amplitude Coupling as a Mechanism for Temporal Orienting of Attention in Childhood. J Cogn Neurosci. 2018; 30(4): 594-602.

PubMed Abstract | Publisher Full Tex

Mento G, Tarantino V, Sarlo M, et al.: Automatic temporal expectancy: a highdensity event-related potential study. PLOS One. 2013; 8(5): e62896.

PubMed Abstract | Publisher Full Text | Free Full Text

Mento G, Tarantino V, Vallesi A, et al.: Spatiotemporal neurodynamics underlying internally and externally driven temporal prediction: a high spatial resolution ERP study. J Cogn Neurosci. 2015; 27(3): 425-39.

PubMed Abstract | Publisher Full Tex

Mento G, Valenza E: Spatiotemporal neurodynamics of automatic temporal expectancy in 9-month old infants. Sci Rep. 2016; 6: 36525.

PubMed Abstract | Publisher Full Text | Free Full Text

Mento G, Vallesi A: Spatiotemporally dissociable neural signatures for generating and updating expectation over time in children: A High DensityERP study. Dev Cogn Neurosci. 2016; 19: 98-106.

PubMed Abstract | Publisher Full Tex

Miniussi C, Wilding EL, Coull JT, et al.: Orienting attention in time. Modulation of brain potentials. Brain. 1999; 122(Pt 8): 1507-18.

PubMled Abstract | Publisher Full Tex

Mossbridge J, Tressoldi PE, Utts J: Predictive physiological anticipation preceding seemingly unpredictable stimuli: a meta-analysis. Front Psychol. 2012; 3: 390.

PubMed Abstract | Publisher Full Text | Free Full Text

Mossbridge J, Tressoldi PE, Utts J, et al.: Predicting the unpredictable: critical analysis and practical implications of predictive anticipatory activity. Front Hum Neurosci. 2014; 8: 146 .

PubMed Abstract | Publisher Full Text | Free Full Text

Oostenveld R, Fries P, Maris E, et al.: FieldTrip: Open source software for advanced analysis of MEG, EEG, and invasive electrophysiological data. Comput Intell Neurosci. 2011; 2011: 156869.

PubMed Abstract | Publisher Full Text | Free Full Text

Pouget A, Beck JM, Ma WJ, et al:: Probabilistic brains: knowns and unknowns. Nat Neurosci. 2013; 16(9): 1170-8.

PubMed Abstract | Publisher Full Text | Free Full Text

Radin DI, Vieten C, Michel L, et al.: Electrocortical activity prior to unpredictable stimuli in meditators and nonmeditators. Explore (NY). 2011; 7(5): 286-299.

PubMed Abstract | Publisher Full Text

Rebert CS, Tecce JJ: A summary of CNV and reaction time. Electroencephalogr Clin Neurophysiol. 1973; 33: 173-178.

Reznik B, Aharonov Y: Time-symmetric formulation of quantum mechanics. Phys Rev A. 1995; 52(4): 2538-2550.

PubMed Abstract | Publisher Full Text

Ritchie SJ, Wiseman R, French CC: Failing the future: three unsuccessful attempts to replicate Bem's 'retroactive facilitation of recall' effect. PLOS One. 2012; 7(3): e33423.

PubMed Abstract | Publisher Full Text | Free Full Text

Stevenson RA, James TW: Affective auditory stimuli: characterization of the International Affective Digitized Sounds (IADS) by discrete emotional categories. Behav Res Methods. 2008; 40(1): 315-321.

PubMed Abstract | Publisher Full Text

Stone JV: Independent component analysis: an introduction. Trends Cogn Sci. 2002; 6(2): 59-64

PubMed Abstract | Publisher Full Tex

Tadel F, Baillet S, Mosher JC, et al: Brainstorm: a user-friendly application for MEG/EEG analysis. Comput Intell Neurosci. 2011; 2011: 879716 .

PubMed Abstract | Publisher Full Text | Free Full Text

Tottenham N, Tanaka JW, Leon AC, et al.: The NimStim set of facial

expressions: judgments from untrained research participants. Psychiatry Res. 2009; $168(3)$ : 242-249.

PubMed Abstract | Publisher Full Text | Free Full Text

Tressoldi P, Duma GM, Mento G: EEG anticipation of random high and low arousal faces and sounds. figshare. Dataset. 2018.

http://www.doi.org/10.6084/m9.figshare.6874871.v4

Tressoldi PE, Maier MA, Buechner VL, et al:: A macroscopic violation of nosignaling in time inequalities? How to test temporal entanglement with behavioral observables. Front Psychol. 2015; 6: 1061.

PubMed Abstract | Publisher Full Text | Free Full Text

Trillenberg $\mathrm{P}$, Verleger $\mathrm{R}$, Wascher $\mathrm{E}$, et al:: $\mathrm{CNV}$ and temporal uncertainty with 'ageing' and 'non-ageing' S1-S2 intervals. Clin Neurophysiol. 2000; 111(7): 1216-1226.

PubMed Abstract | Publisher Full Text

Wagenmakers EJ, Wetzels R, Borsboom D, et al:: Why psychologists must change the way they analyze their data: the case of psi: comment on Bem (2011). 2011; 1(7): 1216-1226.

Walter WG, Cooper R, Aldridge VJ, et al: Contingent Negative Variation: an Electric Sign of Sensorimotor Association and Expectancy in the Human Brain Nature 1964; 230: 380-384.

PubMed Abstract | Publisher Full Text 


\section{Open Peer Review}

\section{Current Peer Review Status:}

\section{Version 1}

Reviewer Report 19 November 2019

https://doi.org/10.5256/f1000research.22279.r54053

(C) 2019 Zheng $\mathbf{X}$ et al. This is an open access peer review report distributed under the terms of the Creative Commons Attribution License, which permits unrestricted use, distribution, and reproduction in any medium, provided the original work is properly cited.

\section{Xifu Zheng}

School of Psychology, South China Normal University, Guangzhou, China

Jingchu $\mathrm{Hu}$

South China Normal University, Guangzhou, China

The manuscript "EEG anticipation of random high and low arousal faces and sounds" reports results from a study involving anticipatory activity, wherein sensory characteristic and arousal level are experimentally manipulated. By using a machine learning algorithm (Support Vector Machine), they found a significantly larger amplitude in occipital cluster of electrodes before the presentation of faces with respect to sounds, along with a larger amplitude in the right auditory cortex before the presentation of sounds with respect to faces. The study is well designed and well written; the results are interesting and add insight to the ongoing effort to understand the sources of anticipatory brain activity. In addition, the authors adopt open science practices, which increase the level and quality of reporting. There are a few minor issues to address:

1. The authors should report exact number of participants in last paragraph of their introduction, instead of "a group of healthy volunteers".

2. The sample size is somehow small, and is not consistent with what they declared in the OSF, the reviewer believe 6 more subject will increase the power, and it's not that difficult to have 6 more.

Is the work clearly and accurately presented and does it cite the current literature? Yes

Is the study design appropriate and is the work technically sound?

Yes

Are sufficient details of methods and analysis provided to allow replication by others? Yes

If applicable, is the statistical analysis and its interpretation appropriate? 
Yes

Are all the source data underlying the results available to ensure full reproducibility? Yes

Are the conclusions drawn adequately supported by the results?

Yes

Competing Interests: No competing interests were disclosed.

Reviewer Expertise: Affective neuroscience

We confirm that we have read this submission and believe that we have an appropriate level of expertise to confirm that it is of an acceptable scientific standard.

Author Response 28 Nov 2019

Patrizio Tressoldi, Università di Padova, Padova, Italy

1. The authors should report exact number of participants in last paragraph of their introduction,

instead of "a group of healthy volunteers".

Reply: We thank the reviewer for the suggestion we added this information in the new version of the manuscript.

2. The sample size is somehow small, and is not consistent with what they declared in the OSF, the reviewer believe 6 more subject will increase the power, and it's not that difficult to have 6 more.

Reply: Unfortunately, in this moment we are not able to increase the sample size due to laboratory and technical issue. However, with respect to the preregistered statistical power, with the final number of participants it reduced from .90 to .82 which is still an acceptable level. Furthermore, we are planning a multicentric study, involving other labs to run a confirmatory study, based on the present results.

Competing Interests: No competing interests were disclosed.

Reviewer Report 18 October 2019

https://doi.org/10.5256/f1000research.22279.r54538

(C) 2019 Debruille J. This is an open access peer review report distributed under the terms of the Creative Commons Attribution License, which permits unrestricted use, distribution, and reproduction in any medium, provided the original work is properly cited. 


\section{J. Bruno Debruille \\ Department of Neuroscience, McGill University, Montreal, QC, Canada}

For this first round of review, the first thing to do is to add the ERPs elicited by the cross from the time the baseline started to be computed up to the end of the ERPs elicited by the stimuli for all experimental conditions.

The type of electrodes (which material?) and the characteristics of the amps and their band pass have to be included in the method section as well as the gain used. Then, the digital band pass used to process the EEG have to be added (with the details about the cut-offs).

Given that the CNV is usually maximal at central or fronto-central electrode sites, data will first have to be re-referenced to one (or two linked) electrodes that are as far as possible from these sites, in order to boost CNV amplitudes and see details. This might also boost effect sizes and give simple statistical analyses a chance to find CNV differences.

These ERPs should be in a figure including a sufficient number of channels (e.g, 20) to give a general idea of the scalp distribution of every component. This will allow for a comparison of the CNV activity found with those found in prior CNV studies.

In this reprocessing, bad channels should not be removed and reconstructed.

Given the extremely high scientific stakes of this study, a simple and FULLY reproducible technique should be used as a strat (even ICA is not fully replicable, its results vary a bit). Any fancy technic should be removed first so that readers can have an idea of the ERPs and CNV obtained.

To do the reprocessing, I suggest to focus on the 20 channels mentioned above. Each time one channel is bad in an EEG epoch: the whole trial should be suppressed. Please give the trial selection criteria (max voltage, flat line...).

Then, please mention the average number of trials that had to be rejected for each experimental condition and the standard deviation across participants.

I will be glad to review the study again and will provide new comments fast according to new results.

Is the work clearly and accurately presented and does it cite the current literature? Partly

Is the study design appropriate and is the work technically sound? Partly

Are sufficient details of methods and analysis provided to allow replication by others? Partly

If applicable, is the statistical analysis and its interpretation appropriate? Partly 
Are all the source data underlying the results available to ensure full reproducibility? Partly

Are the conclusions drawn adequately supported by the results?

Partly

Competing Interests: No competing interests were disclosed.

Reviewer Expertise: Event-related brain potentials and cognition

I confirm that I have read this submission and believe that I have an appropriate level of expertise to state that I do not consider it to be of an acceptable scientific standard, for reasons outlined above.

Author Response 28 Nov 2019

Patrizio Tressoldi, Università di Padova, Padova, Italy

We are very grateful to dr. Debruille for his insightful comments and suggestions. In the current revision we tried to address, to the best of our possibility, most of the issues he raised.

Specifically, we provided additional data and pictures by plotting averaged waveforms from a restricted number of electrodes $(n=19)$ to allow an easy visual inspection of the results. These data have been added as Supplementary Materials available at:

https://doi.org/10.6084/m9.figshare.6874871.v5. Moreover, to provide the reviewer with an easy comparison of the waveform pattern with previous CNV literature, we reported a plot of the whole epoch waveforms by using a linked-mastoid reference (see Figure $1 \mathrm{~b}$ in the Supplementary Materials).

Notwithstanding this, we would like to point out that the dataset was not re-analyzed according to what suggested by the reviewer. In the below point-by-point letter we thoroughly explain the rationale of our choice.

Reply to the reviewer are provided in italic.

1. For this first round of review, the first thing to do is to add the ERPs elicited by the cross from the time baseline started to be computed up to the end of the ERPs elicited by the stimuli for all experimental conditions.

A: We are happy to provide the ERPS of all the conditions starting from the fixation cross as suggested. To make easy the visual inspection of the waveforms we selected 19 electrodes according to the 10:20 system. The new pictures show the comparison between the visual and auditory conditions separately for the active and the passive task.

2.The type of electrodes (which material?) and the characteristics of the amps and their band pass have to be included in the method section as well as the gain used. Then, the digital band pass used to process the EEG have to be added (with the details about the cut-offs).

A: We thank the reviewer for the suggestion, we added the information required in the Method section of the manuscript.

3.Given that the CNV is usually maximal at central or frontocentral electrode sites, data will first have to be 
rereferenced to one (or two linked) electrodes that are as far as possible from these sites, in order toboost $C$ analyses a chance to find CNV differences.

A: We understand the reviewer's point and we thank him for the opportunity to better explain the choice of using an average reference. When using high-density EEG system, the average reference is the gold standard for referencing, as explained by Michel, C. M., Koenig, T., Brandeis, D., Gianotti, L. R., \& Wackermann, J. (2009). In fact, there is not an electrical inactive point on the scalp, neither the mastoids. However, as stated by these authors "[...] the properties of the EEG forward solution are such that for any source, the voltage integral across the entire head surface is zero. If we could cover the entire head with a sufficient number of electrode, we could approximate this voltage integral by the sum of the measurements at all electrodes. Accordingly, we could assume that the sum of the potential differences from all recorded electrodes would be equal to zero. The sum of the potential differences would thus approximate a "correct" zeroreference. Mathematically, this is achieved by using as a reference the average of the measurement at all electrodes. This reference is called, average reference. The validity of the assumption [...] depends on the goodness of coverage of the head by the electrode array" (p35, Michel, C. M., Koenig, T., Brandeis, D., Gianotti, L. R., \& Wackermann, J. (Eds.). (2009). Electrical neuroimaging. Cambridge University Press).

Based on this statement we could assume a that a good coverage of the scalp, as the one provided by an array of 128 geodesic system, would justify the application of the average reference. Moreover, several papers by both our and other groups have been already published with the use of the average reference successfully reporting CNV task-dependent modulations (Mento; Tarantino, Vallesi, Bisiacchi, 2015; Mento and Vallesi, 2016; Mento, 2017; Jang, Jones, Milne, Wilson, Lee, 2016). Furthermore, considering also the statistical approach we used, which includes a whole-scalp explorative analysis,

4.These ERPs should be in a figure including a sufficient number of channels $(e . g, 20)$ to give a general idea of the scalp distribution of every component. This will allow for a comparison of the CNV activity found with those found in prior CNV studies.

In this reprocessing, bad channels should not be removed and reconstructed.

Given the extremely high scientific stakes of this study, a simple and FULLY reproducible technique should be used as a strat (even ICA is not fully replicable, its results vary a bit). Any fancy technic should be removed first so that readers can have an idea of the ERPs and CNV obtained. To do the reprocessing, I suggest to focus on the 20 channels mentioned above. Each time one channel is bad in an EEG epoch: the whole trial should be suppressed. Please give the trial selection criteria ( $\max$ voltage, flat line...).

Then, please mention the average number of trials that had to be rejected for each experimental condition and the standard deviation across participants.

A: We agree that ICA manual components removal may change a bit across experimenters, nonetheless it is a reliable procedure commonly accepted by ERP scientific community, as stated by several studies (Mennes, M., Wouters, H., Vanrumste, B., Lagae, L., \& Stiers, P. (2010).

Validation of ICA as a tool to remove eye movement artifacts from EEG/ERP. Psychophysiology, 47(6), 1142-1150; Jung, T. P., Humphries, C., Lee, T. W., Makeig, S., McKeown, M. J., Iragui, V., \& Sejnowski, T. J. (1998). Extended ICA removes artifacts from electroencephalographic recordings. In Advances in neural information processing systems (pp. 894-900); Li, R., \& Principe, J. C. (2006, 
August). Blinking artifact removal in cognitive EEG data using ICA. In 2006 International Conference of the IEEE Engineering in Medicine and Biology Society (pp. 5273-5276). IEEE. Drisdelle, B. L., Aubin, S., \& Jolicoeur, P. (2017). Dealing with ocular artifacts on lateralized ERPs in studies of visual-spatial attention and memory: ICA correction versus epoch rejection. Psychophysiology, 54(1), 83-99.). Moreover, in our case the variability intrinsic to this procedure has been sensibly reduced since we exclusively targeted eye-, muscle- and heartbeatrelated artifacts. As an additional consideration, with the High-Density EEG, the ICA is the best way to correct artifacts specially in the case of eye-related artifacts. In fact, due to the high number of electrodes around the eyes (around 20), their activity is very spread across frontal electrodes, especially if considering that an average reference usually magnifies dipolar scalp configuration. This in turn may result in the contamination of posterior electrodes, that could show residual eye-related artefactual activity overlapped to the CNV.

It follows that simply applying a threshold would reject lots of trials, seriously undermining the signal-to-noise ratio and underpowering the statistical comparison.

In other words, while we in principle agree with the reviewer that an artifact correction procedure may introduce some variability in the reproducibility of the results, at the same time we believe that a too rigid cleaning procedure based on a threshold-based artifact rejection may 1) not being effective in eye-related artifact cleaning and 2) cause an excessive lack of statistical power due to a dramatic reduction of the signal-to-noise ratio resulting from a massive trial rejection. For this reason, we still believe that ICA is the best approach in the High-Density EEG recording. Anyway, in order to assure replicability, we could provide upon request a list of the components removed for each subject.

Concerning bad channels interpolation removal and reconstruction, we would assure the reviewer that this is a standard method of HD-EEG preprocessing, also suggested in the Makoto's pipeline for EEG analysis and in the Prepipeline toolbox, (https://vis/ab.github.io/EEG-Clean-Tools/ ), which is a method to standardize EEG recordings across experimenters. Given that, it is not possible to avoid bad channel identification and correction. We also want to specify that the number of interpolated electrodes was very low (around 8-10 electrodes over 128) and was restricted to the external belt ones surrounding the ears, as a possible aspect of the geodesic sensor net is that those electrodes sometime do not adhere perfectly to the scalp.

Concerning the mean number of trials and standard deviation for each experimental condition across participants we added this information in the manuscript, see Table 1.

Finally, we would like to add that we are really pleased that our manuscript found the reviewer's interest. Nonetheless, we desire to underline that the main purpose of the paper is to bring the scientific community attention to the possibility that random anticipatory effect may be actual, in order to generate independent replication of our findings.

Competing Interests: I'm the corresponding author 
The benefits of publishing with F1000Research:

- Your article is published within days, with no editorial bias

- You can publish traditional articles, null/negative results, case reports, data notes and more

- The peer review process is transparent and collaborative

- Your article is indexed in PubMed after passing peer review

- Dedicated customer support at every stage

For pre-submission enquiries, contact research@f1000.com 